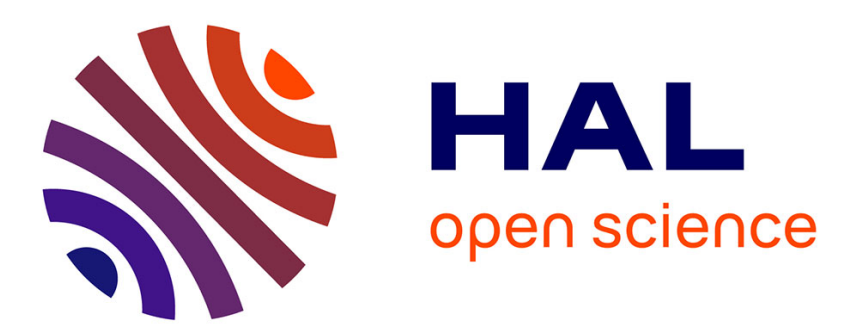

\title{
Hamiltonian Decompositions of Graphs, Directed Graphs and Hypergraphs
}

\author{
Jean-Claude Bermond
}

\section{To cite this version:}

Jean-Claude Bermond. Hamiltonian Decompositions of Graphs, Directed Graphs and Hypergraphs. Annals of Discrete Mathematics, 1978, 3, pp.21-28. 10.1016/S0167-5060(08)70494-1 . hal-02321683

\section{HAL Id: hal-02321683 \\ https://hal.science/hal-02321683}

Submitted on 21 Oct 2019

HAL is a multi-disciplinary open access archive for the deposit and dissemination of scientific research documents, whether they are published or not. The documents may come from teaching and research institutions in France or abroad, or from public or private research centers.
L'archive ouverte pluridisciplinaire HAL, est destinée au dépôt et à la diffusion de documents scientifiques de niveau recherche, publiés ou non, émanant des établissements d'enseignement et de recherche français ou étrangers, des laboratoires publics ou privés. 


\title{
HAMILTONIAN DECOMPOSITIONS OF GRAPHS, DIRECTED GRAPHS AND HYPERGRAPHS
}

\author{
J.-C. BERMOND \\ C.M.S., 54 Bd. Raspail, 75006 Paris, Cédex 06. France
}

\section{Definitions}

Definitions not given here can be found in [3].

We will say that a graph $G$ (undirected or directed) can be decomposed into Hamiltonian cycles or paths if we can partition its edges (arcs in the directed case) into hamiltonian cycles or paths (directed cycles or directed paths in the directed case). Our notation is as follows:

$K_{n}$ - the complete graph on $n$ vertices;

$K_{n}^{*}$ - the complete symmetric directed graph on $n$ vertices;

$K_{r \times n}$ - the complete $r$-partite graph whose vertex set is the disjoint union of $r$ sets of $n$ elements, two vertices being joined iff they belong to two different sets;

$C_{r}$ (resp. $\vec{C}_{r}$ ) - a cycle (resp. directed cycle) of length $r$;

$S_{n}$ - an independent set of $n$ vertices;

$G_{1} \times G_{2}$ - the cartesian sum (also called product) of two graphs $G_{1}=\left(X_{1}, E_{1}\right)$ and $G_{2}=\left(X_{2}, E_{2}\right)$ is the graph with vertex set $X_{1} \times X_{2}$ in which $\left(x_{1}, x_{2}\right)$ is joined to $\left(y_{1}, y_{2}\right)$ whenever $x_{1}=y_{1}$ and $x_{2}$ is joined to $y_{2}$ in $G_{2}$, or $x_{2}=y_{2}$ and $x_{1}$ is joined to $y_{1}$ in $G_{1}$;

$G_{1} \otimes G_{2}$ - the lexicographic product (also called composition) of two graphs $G_{1}$ and $G_{2}$ is the graph with vertex set $X_{1} \times X_{2}$ in which $\left(x_{1}, x_{2}\right)$ is joined to $\left(y_{1}, y_{2}\right)$ whenever $x_{1}$ is joined to $y_{1}$ in $G_{1}$ or $x_{1}=y_{1}$ and $x_{2}$ is joined to $y_{2}$ in $G_{2}$ :

$G_{1} \cdot G_{2}$ - the cartesian product (also called conjunction) of two graphs $G_{1}$ and $G_{2}$ is the graph with vertex set $X_{1} \times X_{2}$ in which $\left(x_{1}, x_{2}\right)$ is joined to $\left(y_{1}, y_{2}\right)$ whenever $x_{1}$ is joined to $y_{1}$ in $G_{1}$ and $x_{2}$ is joined to $y_{2}$ in $G_{2}$.

\section{Hamiltonian decompositions of graphs}

The first two results are folklore.

1.1. Theorem. $K_{2 n}$ can be decomposed into $n$ hamiltonian paths.

1.2. Theorem. $K_{2 n+1}$ can be decomposed into $n$ hamiltonian cycles and $K_{2 n+2}$ can be decomposed into $n$ hamiltonian cycles and a perfect matching (or 1-factor). 
1.3. Theorem (Auerbach and Laskar [2]). $K_{r \times n}$ can be decomposed into hamiltonian cycles iff $n(r-1)$ is even. If $n(r-1)$ is odd $K_{r \times n}$ can be decomposed into hamiltonian cycles and a perfect matching.

We will now give a survey of hamiltonian decompositions of the three products defined in Section 0 and pose some problems concerning them.

1.4. Theorem (Kotzig [12]). $C_{r} \times C_{n}$ can be decomposed into 2 hamiltonian cycles.

1.5. Remark. The case $r=n$ is also proved in Myers [15].

1.6. Corollary. If each of $G_{1}$ and $G_{2}$ can be decomposed into $p$ hamiltonian cycles, then $G_{1} \times G_{2}$ can be decomposed into $2 p$ hamiltonian cycles.

1.7. Theorem (Myers [15]). $K_{n} \times K_{n}$ can be ciecomposed into $(n-1)$ hamiltonian cycles.

1.8. Conjecture (Kotzig .[12]). $C_{r} \times C_{n} \times C_{m}$ can be decomposed into hamiltonian cycles.

1.9. Remarks on Conjecture 1.8. G. Koester (personal communication, 1977) has proved that $C_{4} \times C_{4} \times C_{4}$ can be decomposed into hamiltonian cycles. In fact he informed me that the problem of the existence of a decomposition of $C_{4} \times C_{4} \times$ $\cdots \times C_{4}$ ( $n$ times) was posed by Ringel [16, Problem 2] as the existence of a decomposition of the $2 n$-cube ( $2 n$-dimensional Würfel) into hamiltonian cycles. Ringel [16] proved this conjecture for $n$ a power of 2: this also follows from Theorem 1.4 with $r=n=4$ and Corollary 1.6.

Very recently I learned that the existence of a decomposition of $C_{3} \times C_{3} \times C_{3}$ into hamiltonian cycles was proved by M. Foregger (personal communication of R. Brualdi, 1977).

1.10. Conjecture. $K_{m} \times K_{n}$ can be decomposed into $\frac{1}{2}(n+m-2)$ hamiltonian cycles iff $n+m$ is even and into $\frac{1}{2}(n+m-3)$ hamiltonian cycles and a perfect matching if $n+m$ is odd.

1.11. Conjecture. If $G_{1}$ can be decomposed into $p_{1}$ hamiltonian cycles and if $G_{2}$ can be decomposed into $p_{2}$ hamiltonian cycles, then $G_{1} \times G_{2}$ can be decomposed into $p_{1}+p_{2}$ hamiltonian cycles.

1.12. Theorem (Laskar [13]). $C_{r} \otimes S_{n}$ can be decomposed into $n$ hamiltonian cycles.

1.13. Remark. Theorem 1.12 can be used to give a short proof of Theorem 1.3 since 
$K_{r \times n}=K_{r} \otimes S_{n}$. If $r$ is odd, then by Theorem $1.2 K_{r}$ can be decomposed into hamiltonian cycles, that is $K_{r}=\bigcup_{i} C_{r}^{(i)}$ (where $\bigcup$ means the edge-disjoint union and $1 \leqslant i \leqslant r)$ and $K_{r \times n}=\bigcup_{i} C_{t}^{(i)} \otimes S_{n}=\bigcup_{i, j} C_{r n}^{(i, j)}$ by Theorem 1.12. If $r$ is even, then $K_{\frac{1}{2} r \times 2}$ is the graph obtained from $K_{r}$ by deleting a perfect matching and so can be decomposed into hamiltonian cycles by Theorem 1.2. Finally $K_{r \times 2 p}=$ $\left(K_{r \times 2}\right) \otimes S_{p}=\bigcup_{i} C_{2 r}^{(i)} \otimes S_{p}=\bigcup_{i, j} C_{2 r p}^{(i, j)}$. And thus if $n(r-1)$ is even $K_{r \times n}$ can be decomposed into hamiltonian cycles.

1.14. Theorem (Laskar [13]). $C_{r} \otimes C_{n}$ can be decomposed into $n+1$ hamiltonian cycles if $n$ is odd or $r$ is even.

1.15. Conjecture. $C_{r} \otimes C_{n}$ can always be decomposed into $n+1$ hamiltonian cycles.

1.16. Conjecture. If $G_{1}$ can be decomposed into $p_{1}$ hamiltonian cycles and if $G_{2}$ can be decomposed into $p_{2}$ hamiltonian cycles, then $G_{1} \otimes G_{2}$ can be decomposed into $p_{1} n_{2}+p_{2}$ hamiltonian cycles (where $n_{2}$ is the number of vertices of $G_{2}$ ).

1.17. Remark. I can prove that Conjecture 1.15 implies the truth of Conjecture 1.16 for $p_{1} \geqslant p_{2}$.

1.18. Theorem. $C_{r} \cdot C_{n}$ can be decomposed into 2 hamiltonian cycles.

Proof. We can suppose $r \geqslant n$; let the vertex set be $\mathbb{Z}_{r} \times \mathbb{Z}_{n}$ (where $\mathbb{Z}_{n}$ denotes the additive group of residues $\bmod n)$. Then two hamiltonian cycles are $x_{0}, x_{1}, \ldots, x_{r n-1}$ and $y_{0}, y_{1}, \ldots, y_{r n-1}$ where $x_{i n+j}=(i+j, j)$ and $y_{i n+j}=(-i-j, j)$, $0 \leqslant i \leqslant r-1,0 \leqslant j \leqslant n-1$.

1.19. Corollary. If $G_{1}$ and $G_{2}$ can be decomposed into hamiltonian cycles, then $G_{1} \cdot G_{2}$ also can be decomposed into hamiltonian cycles.

Proof. This follows from the distributivity of the product - with respect to the edge disjoint union of graphs (a property not holding for the cartesian sum and the lexicographic product).

1.20. Many other problems similar to those above can be considered; in particular we can consider decompositions into cycles of given length (see [6]). We mention also that Huang and Rosa [9] have considered "orthogonal" hamiltonian decompositions, and finally we give the following conjecture of Kotzig [11].

1.21. Conjecture (Kotzig [11]). $K_{2 n}$ can be decomposed into perfect matchings, i.e. has a 1 factorisation, in such a manner that the unign of any two perfect matchings is a hamiltonian cycle. 
Partial results have been obtained on this problem, see for example Anderson [1].

\section{Hamiltonian decompositions of directed graphs}

The same problems can be asked for the directed graphs, but they are more difficult. Many of the known results are obtained in the following easy way; associate with a hamiltonian decomposition of $G$ a hamiltonian decomposition of the directed graph $G^{*}$ (obtained from $G$ by associating to each edge of $G$ two opposite arcs) by associating with each hamiltonian cycle two opposite directed hamiltonian cycles. For example Theorem 1.2 gives the following.

2.1. Theorem. $K_{2 n+1}^{*}$ can be decomposed into $2 n$ directed hamiltonian cycles.

The problem of the existence of a hamiltonian decomposition of $K_{n}^{*}$ has been solved only recently.

2.2. Theorem (Tillson [17]). If $2 n \geqslant 8$, then $K_{2 n}^{*}$ can be decomposed into $2 n-1$ directed hamiltonian cycles.

2.3. For $2 n=4$ and $2 n=6$, such a decomposition is impossible. The problem seems to have been asked first by Strauss for hamiltonian paths (see Mendelsohn [14]). In [14] Mendelsohn showed how the existence of sequenceable groups implies the existence of a hamiltonian decomposition of $K_{2 n}^{*}$ and that gives the result for $2 n=22$ [14], 28 [10], 40, 56, 58 [18]. By computer the existence of a hamiltonian decomposition of $K_{2 n}^{*}$ for $8 \leqslant 2 n \leqslant 18$ was obtained (see [5]). With Faber we proposed Theorem 2.2 as conjecture in [5] and [4]. After that A. Bouchet (personal communication, 1976) showed that if $K_{2 n}^{*}$ can be decomposed into directed hamiltonian cycles, then so can $K_{4 n-2}^{*}$.

2.4. Conjecture. One can easily ask many other problems, for example the directed versions of the results or problems of Section 1. But there are also problems peculiar to the directed case like Kelly's conjecture that every regular tournament can be decomposed into directed hamiltonian cycles.

\section{Hamiltonian decompositions of hypergraphs}

3.1. For hypergraphs the number of problems grows quickly, because one can give different definitions of a hamiltonian cycle. I will restrict myself to a definition and a problem considered in [7]. If $H$ is a hypergraph with $n$ vertices then a hamiltonian cycle is a sequence $x_{1} E_{1} x_{2} \cdots x_{i} E_{i} x_{i+1} \cdots x_{n} E_{n} x_{1}$ such that 
(i) the $n$ vertices $x_{i}$ are all different (and thus are the $n$ vertices of the hypergraph),

(ii) the $n$ edges $E_{i}$ are all different,

(iii) $\left\{x_{i}, x_{i+1}\right\} \subset E_{i}(1 \leqslant i \leqslant n-1)$ and $\left\{x_{1}, x_{n}\right\} \subset E_{n}$.

Let $K_{n}^{h}$ denote the complete $h$-uniform hypergraph; its edges are all the $h$-subsets of a set $X$ of cardinality $n$.

In [7] we conjectured that $K_{n}^{h}$ can be decomposed into hamiltonian cycles if and only if $\left(\begin{array}{l}n \\ h\end{array}\right) / n$ is an integer and proved this conjecture for $n$ a prime.

Here I want to prove two theorems concerning the case $h=3$.

3.2. Theorem. If $K_{n}^{3}$ can be decomposed into hamiltonian cycles then $K_{2 n}^{3}$ also can be decomposed into hamiltonian cycles.

Proof. (This proof was obtained with D. Sotteau.) In order to shorten the writing, I will write a hamiltonian cycle as $E_{1}, \ldots E_{i}, \ldots, E_{n}$ where $E_{i}=\left(x_{i}, y_{i}, x_{i+1}\right)$. Let the vertex set of $K_{2 n}^{3}$ be $X \cup X^{\prime}$ with $|X|=\left|X^{\prime}\right|=n$. With each hamiltonian cycle of the decomposition of $K_{n}^{3}$ we associate 4 hamiltonian cycles of $K_{2 n}^{3}$ in the following manner:

(i) if $n$ is even, we associate with $\left(x_{1} y_{1} x_{2}\right)\left(x_{2} y_{2} x_{3}\right), \ldots,\left(x_{n 1} y_{1 n} x_{1}\right)$ the following

$$
\begin{array}{r}
\left(x_{1} y_{1} x_{2}\right)\left(x_{2} y_{2} x_{3}\right) \cdots\left(x_{n-1} y_{n-1} x_{n}\right)\left(x_{n} y_{n} x_{1}^{\prime}\right) \\
\left(x_{1}^{\prime} y_{1} x_{2}^{\prime}\right) \cdots\left(x_{n-1}^{\prime} y_{n-1} x_{n}^{\prime}\right)\left(x_{n}^{\prime} y_{n} x_{1}\right), \\
\left(x_{1} y_{1} x_{2}^{\prime}\right)\left(x_{2}^{\prime} y_{2} x_{3}\right)\left(x_{3} y_{3} x_{4}^{\prime}\right) \cdots\left(x_{n-1} y_{n-1} x_{n}^{\prime}\right)\left(x_{n}^{\prime} y_{n} x_{1}^{\prime}\right) \\
\left(x_{1}^{\prime} y_{1} x_{2}\right)\left(x_{2} y_{2} x_{3}^{\prime}\right) \cdots\left(x_{n-1}^{\prime} y_{n-1} x_{n}\right)\left(x_{n} y_{n} x_{1}\right),
\end{array}
$$

and the two cycles obtained by exchanging the vertices of $X$ and those of $X^{\prime}$ :

(ii) if $n$ is odd we associate with $\left(x_{1} y_{1} x_{2}\right)\left(x_{2} y_{2} x_{3}\right), \ldots,\left(x_{n} y_{n} x_{1}\right)$ the following:

$$
\begin{gathered}
\left(x_{1} y_{1} x_{2}\right)\left(x_{2} y_{2} x_{3}\right) \cdots\left(x_{n-2} y_{n-2} x_{n-1}\right)\left(x_{n-1} y_{n-1}^{\prime} x_{n}^{\prime}\right)\left(x_{n}^{\prime} y_{n} x_{1}^{\prime}\right) \\
\left(x_{1}^{\prime} y_{1} x_{2}^{\prime}\right)\left(x_{2}^{\prime} y_{2} x_{3}^{\prime}\right) \cdots\left(x_{n-2}^{\prime} y_{n-2} x_{n-1}^{\prime}\right)\left(x_{n-1}^{\prime} y_{n-1}^{\prime} x_{n}\right)\left(x_{n} y_{n} x_{1}\right), \\
\left(x_{1}^{\prime} y_{1}^{\prime} x_{2}^{\prime}\right)\left(x_{2}^{\prime} y_{2}^{\prime} x_{3}^{\prime}\right) \cdots\left(x_{n-2}^{\prime} y_{n-2}^{\prime} x_{n-1}^{\prime}\right)\left(x_{n-1}^{\prime} y_{n-1}^{\prime} x_{n}^{\prime}\right)\left(x_{n}^{\prime} y_{n}^{\prime} x_{1}\right) \\
\quad\left(x_{1} y_{1}^{\prime} x_{2}\right)\left(x_{2} y_{2}^{\prime} x_{3}\right) \cdots\left(x_{n-2} y_{n-2}^{\prime} x_{n-1}\right)\left(x_{n-1} y_{n-1}^{\prime} x_{n}\right)\left(x_{n} y_{n}^{\prime} x_{1}^{\prime}\right), \\
\left(x_{1} y_{1} x_{2}^{\prime}\right)\left(x_{2}^{\prime} y_{2} x_{3}\right) \cdots\left(x_{n-2} y_{n-2} x_{n-1}^{\prime}\right)\left(x_{n-1}^{\prime} y_{n-1} x_{n}\right)\left(x_{n} y_{n} x_{1}^{\prime}\right) \\
\left(x_{1}^{\prime} y_{1} x_{2}\right)\left(x_{2} y_{2} x_{3}^{\prime}\right) \cdots\left(x_{n-2}^{\prime} y_{n-2} x_{n-1}\right)\left(x_{n-1} y_{n-1} x_{n}^{\prime}\right)\left(x_{n}^{\prime} y_{n} x_{1}\right),
\end{gathered}
$$

and

$$
\begin{aligned}
& \left(x_{1}^{\prime} y_{1}^{\prime} x_{2}\right)\left(x_{2} y_{2}^{\prime} x_{3}^{\prime}\right) \cdots\left(x_{n-2}^{\prime} y_{n-2}^{\prime} x_{n-1}\right)\left(x_{n-1} y_{n-1} x_{n}\right)\left(x_{n} y_{n}^{\prime} x_{1}\right) \\
& \left(x_{1} y_{1}^{\prime} x_{2}^{\prime}\right)\left(x_{2}^{\prime} y_{2}^{\prime} x_{3}\right) \cdots\left(x_{n-2} y_{n-2}^{\prime} x_{n-1}^{\prime}\right)\left(x_{n-1}^{\prime} y_{n-1} x_{n}^{\prime}\right)\left(x_{n}^{\prime} y_{n}^{\prime} x_{1}^{\prime}\right) .
\end{aligned}
$$

Thus we have obtained a decomposition of the edges of $K_{2 n}^{3}$ not of the form $\left(x, x^{\prime}, y\right)$ or $\left(x, x^{\prime}, y^{\prime}\right)$. We will use Theorems 2.1 and 2.2 to decompose these remaining triples. Indeed with the directed hamiltonian cycle $x_{1}, \ldots, x_{n}$ of a 
decomposition of $K_{n}^{*}$ we associate the following hamiltonian cycle of $K_{n}^{3}$ :

$$
\begin{aligned}
& \left(x_{1} x_{1}^{\prime} x_{2}\right)\left(x_{2} x_{2}^{\prime} x_{3}\right) \cdots\left(x_{n-1} x_{n-1}^{\prime} x_{n}\right)\left(x_{n} x_{n}^{\prime} x_{1}^{\prime}\right) \\
& \qquad\left(x_{1}^{\prime} x_{1} x_{2}^{\prime}\right) \cdots\left(x_{n-1}^{\prime} x_{n-1} x_{n}^{\prime}\right)\left(x_{n}^{\prime} x_{n} x_{1}\right) .
\end{aligned}
$$

Thus the proof is complete. One can check that we have found $\left[\frac{4}{6}(n-1)(n-2)\right]+$ $n-1=\frac{1}{6}(2 n-1)(2 n-2)$ hamiltonian cycles in $K_{2 n}^{3}$.

3.3. Theorem. If $n \equiv 2 \bmod (3) K_{n}^{3}$ can be decomposed into hamiltonian cycles.

We are grateful to A.E. Brouwer (personal communication, 1976) for the following idea on which the proof is based.

3.4. A choice design of order $n$ is a system of representatives of the triples of $K_{n}^{3}$ such that:

(i) each point is chosen equally often as a representative;

(ii) among the $n-2$ triples containing a given pair $\{a, b\}, a$ is chosen $\frac{1}{3}(n-2)$ times and $b \frac{1}{3}(n-2)$ times also.

For example, when $n=5$, we have underlined the element chosen.

$$
0 \underline{12} ; 01 \underline{3} ; 014 ; \underline{02} 3 ; 0 \underline{2} 4 ; 03 \underline{4} ; 12 \underline{2} ; 12 \underline{4} ; 134 ; 2 \underline{3} 4 .
$$

3.5. Theorem. A choice design of order $n$ exists if and only if $n \equiv 2(\bmod 3)$.

Proof. The necessary condition is obvious as $\frac{1}{3}(n-2)$ must be an integer. We will prove that the condition is sufficient by induction. Suppose there exists a choice design of order $n$. Let the elements of $K_{n+3}^{3}$ be $\{1,2, \ldots n\} \cup\{\alpha, \beta, \gamma\}$. For a triple of elements of $\{1,2, \ldots, n\}$ we choose the element defined by the choice design of order $n$. For the triples we choose

$$
\begin{array}{cccl}
(i, j, \alpha) & (i, j, \beta) & (i, j, \gamma) & \text { with } i<j, \\
i & j & \gamma & \text { if } i+j \equiv 0(\bmod 3), \\
j & \beta & i & \text { if } i+j \equiv 1(\bmod 3), \\
\alpha & i & j & \text { if } i+j \equiv 2(\bmod 3) .
\end{array}
$$

For the triples we choose

$$
\begin{array}{cccc}
(i, \alpha, \beta) & (i, \alpha, \gamma) & (i, \beta, \gamma) & \\
i & \gamma & \gamma & \text { if } i \equiv 0(\bmod 3), \\
\beta & \alpha & i & \text { if } i \equiv 1(\bmod 3), \\
\alpha & i & \beta & \text { if } i \equiv 2(\bmod 3) .
\end{array}
$$

For the triple $(\alpha, \beta, \gamma)$ we choose $\gamma$.

We leave to the reader the care of checking that we obtain a choice design of order $n+3$; the only non-immediate part is to check property (ii) for the triples containing a pair $(i, \alpha)$ or $(i, \beta)$ or $(i, \gamma)$. 
3.6. Proof of Theorem 3.3. If $n$ is odd, there exists a decomposition of $K_{n}$ into $\frac{1}{2}(n-1)$ hamiltonian cycles. To each of these cycles $\left(x_{1}, x_{2}, \ldots, x_{n}\right)$ we associate the following $\frac{1}{3}(n-2)$ hamiltonian cycles of $K_{n}^{3}:\left(x_{1}, y_{1}^{i}, x_{2}\right)\left(x_{2}, y_{2}^{i}, x_{3}\right) \cdots$ $\left(x_{n-1}, y_{n-1}^{i}, x_{n}\right)\left(x_{n}, y_{n}^{i}, x_{1}\right)$, where $i=1,2, \ldots, \frac{1}{3}(n-2), y_{j}^{i} \neq y_{j}^{k}$ for $i \neq k$ and where the $y_{j}^{i}$ are defined according to the existence of a choice design of order $n$ (by Theorem $3.5)$. The set $\left\{y_{j}^{i}: i=1,2, \ldots, \frac{1}{3}(n-2)\right\}$ consists of the $\frac{1}{3}(n-2)$ elements representatives of the $\frac{1}{3}(n-2)$ triples $\left(x_{j}, x_{j+1}, y\right)$ containing the pair $\left\{x_{j}, x_{j+1}\right\}$ and where neither $x_{j}$ nor $x_{j+1}$ has been chosen. Thus we have constructed $\frac{1}{6}(n-1)(n-2)$ hamiltonian cycles of $K_{n}^{3}$ and it suffices to verify that no triple (edge) appears twice, but that follows from the definition of a choice design of order $n$.

3.7. Example. Let $(0,1,2,3,4)$ and $(0,2,4,1,3)$ be two hamiltonian cycles of $K_{5}$; by using the choice design of the example we obtain the two hamiltonian cycles of $K_{5}^{3}$ :

$$
\begin{aligned}
& (0,3,1)(1,4,2)(2,0,3)(3,1,4)(4,2,0), \\
& (0,1,2)(2,3,4)(4,0,1)(1,2,3)(3,4,0) .
\end{aligned}
$$

If $n$ is even the proof is similar. We use a decomposition of $K_{n}^{*}$ into $n-1$ directed hamiltonian cycles (Theorem 2.2). To each of these directed cycles $\left(x_{1}, x_{2}, \ldots, x_{n}\right)$ we associate $\frac{1}{6}(n-2)$ hamiltonian cycles of $K_{n}^{3}$ :

$$
\left(x_{1}, y_{1}^{i}, x_{2}\right)\left(x_{2}, y_{2}^{i}, x_{3}\right) \cdots\left(x_{n-1}, y_{n-1}^{i}, x_{n}\right)\left(x_{n}, y_{n}^{i}, x_{1}\right) \text {, }
$$

where $i=1,2, \ldots, \frac{1}{6}(n-2) ; \quad y_{j}^{i} \neq y_{j}^{k}$ for $i \neq k$ and where the set $\left\{y_{j}^{i}: i=\right.$ $1,2, \ldots, \frac{1}{6}(n-2)$ is determined as follows. Consider the $\frac{1}{3}(n-2)$ elements representative of the triples $\left(x_{j}, x_{j+1}, y\right)$ containing the pair $\left\{x_{j}, x_{j+1}\right\}$ and where neither $x_{j}$ nor $x_{j+1}$ has been chosen. Then split these elements into two sets of cardinality $\frac{1}{6}(n-2): Y_{\left\{x_{i}, x_{i-1}\right\}}$ and $Y_{\left\{x_{i}, x_{j+1}\right\}}^{\prime}$. Then the set $\left\{y_{j}^{i}: i=1,2, \ldots, \frac{1}{6}(n-2)\right\}$ is either the set $Y_{\left\{x_{i}, x_{j-1}\right\}}$ or $Y_{\left\{x_{j}, x_{i-1}\right\}}^{\prime}$ according as the arc $\left(x_{j}, x_{j+1}\right)$ or the arc $\left(x_{j+1}, x_{j}\right)$ appears in the directed hamiltonian cycle.

\section{Note added in proof}

M.F. Foregger has proved Conjecture 1.8 (Hamiltonian Decompositions of Product of Cycles).

\section{References}

[1] B.A. Anderson, A perfectly arranged room square, Proc. 4th Southeastern Conference on Combinatorics, Graph Theory, and Computing, Utilitas Math. (1973) 141-150.

[2] B. Auerbach and R. Lasker. On decompositions of $r$-partite graphs into edge - disjoint hamiltonian circuits, Discrete Math. 14 (1976) 265-268.

[3] C. Berge. Graphs and Hypergraphs (North-Holland, Amsterdam, 1973). 
[4] J.-C. Bermond and V. Faber, in: Problems of the 5th British Combinatorial Conference, Aberdeen 1975. Utilitas Math. Congressus Numerantium XV (1975). 695.

[5] J.-C. Bermond and V. Faber, Decomposition of the complete directed graph into $k$-circuits, J. Combinatorial Theory, 21(B) (1976) 146-155.

[6] J.-C. Bermond and D. Sotteau, Graph Decompositions and G-designs, Proc. 5th Combinatorial Conference, Aberdeen 1975, Utilitas Math. Congressus Numerantium XV (1975) 53-72.

[7] J.-C. Bermond, A. Germa, M.-C. Heydemann and D. Sotteau, Hypergraphes hamiltoniens, in: Coll. Int. C.N.R.S., Problèmes Combinatoires et Théorie des Graphes, Orsay, 1976.

[8] J. Denes and A.D. Keedwell, Latin squares and their applications. (Akademiai Kiado, Budapest and English University Press, London, 1974).

[9] C. Huang and A. Rosa, On sets of orthogonal hamiltonian circuits, Proc. 2nd Manitoba Conference on Numerical Mathematics, Utilitas Math. Congressus Numerantium VII (1972), 327-332.

[10] A.D. Keedwell, Some problems concerning complete latin squares. Combinatorics, Proc. British Combinatorial Conference, 1973, L.M.S. Notes 13, Cambridge University (1974) 89-96.

[11] A. Kotzig, Problem, Theory of Graphs and Its Applications (Academic Press, New York, 1964) 162 and 63-82.

[12] A. Kotzig, Every cartesian product of two circuits is decomposable into two hamiltonian circuits, Centre de Recherches Mathematiques, Montreal (1973).

[13] R. Laskar, Decomposition of some composite graphs into hamiltonian cycles, Proc. 5th Hungarian Colloquium. Keszthely, 1976, (to appear).

[1+] N.S. Mendelsohn. Hamiltonian decomposition of the complete directed $n$-graph, Theory of Graphs (Akademiai Kiado. Budapest, 1968) 237-2+1.

[15] B.R. Myers, Hamiltonian factorization of the product of a complete graph with itself, Networks 2 (1972) 1-9.

[16] G. Ringel, Über drei kombinatorische Probleme am n-dimensionalen Würfel und Würfelgitter, Abh. Math. Sem. Hamburg 20 (1954) 10-19.

[17] T. Tillson, A hamiltonian decomposition of $K_{2 m}^{*}, 2 m \geqslant 8$, J. Combinatorial Theory (B) (to appear).

[18] L.L. Wang, A test for sequencing a class of finite groups with two generators, Notices Am. Math. Soc. 20 (1973) A632. 